\section{Al-18 THE EFFECT OF BELIMUMAB ON B CELL SELECTION IN HUMAN SLE}

${ }^{1}$ Weiqing Huang, ${ }^{1}$ Tam Quach, ${ }^{1}$ Cosmin Dascalu, 'Zheng Liu, ${ }^{2}$ Tungming Leung, ${ }^{3}$ Jian Han, ${ }^{2}$ Martin Lesser, ${ }^{4}$ Thomas Rothstein, ${ }^{1}$ Richard Furie, ${ }^{1}$ Meggan Mackay, ${ }^{1}$ Cynthia Aranow, ${ }^{1}$ Anne Davidson*. ${ }^{1}$ Center for Autoimmunity and Musculoskeletal and Hematologic Diseases, Feinstein Institute for Medical Research, Manhasset, New York, NY; ${ }^{2}$ Biostatistics Unit, Feinstein Institute for Medical Research, Manhasset, New York, NY; ${ }^{3}$ Hudson Alpha Institute for Biotechnology, Huntsville AL; ${ }^{4}$ Western Michigan University Homer Stryker M.D. School of Medicine, Kalamazoo, MI

\subsection{6/lupus-2018-Ism.18}

Background Belimumab has a therapeutic benefit in active SLE, especially in patients with high titers of anti-dsDNA antibodies. The current study was designed to address whether the profound loss of naïve $\mathrm{B}$ cells in belimumab treated patients is accompanied by a shift in the immunoglobulin repertoire of either mature B cells or plasma cells.

Methods 15 SLE patients who had been continuously treated with belimumab $10 \mathrm{mg} / \mathrm{kg}$ monthly for $>5$ years were matched with 17 SLE controls. 5 SLE patients newly starting on belimumab were studied before and 6 months after drug initiation. B cell phenotyping was performed using flow cytometry. Mature B cells and plasmablasts were sort purified, VH libraries were generated using barcoded primers (iRepertoire) and pooled libraries were sequenced using miSeq. Analyses of unmutated and mutated IgM sequences from mature B cells and all sequences from plasmablasts were performed using customized Perl and R scripts.

Results Phenotyping - novel findings:

1. BAFF regulates the transitional $\mathrm{B}$ cell checkpoint with conservation of transitional type 1 cells and $\approx 90 \%$ loss of transitional type 3 and naïve B cells after chronic belimumab treatment.

2. Neither 'naïve activated' B cells nor CD21 lo B cells subset are preferentially depleted by belimumab.

3. The early increase in CD27+ class switched cells after belimumab treatment is due to an increase in memory B cells rather than B1 cells.

4. After $>5$ years of treatment, class switched memory B cells, B1 B cells and plasmablasts are also substantially depleted.

Next Generation Sequencing of $\mathrm{VH}$ genes:

1. There was no redistribution of $\mathrm{V}, \mathrm{D}$ or $\mathrm{J}$ family usage among unmutated IgM sequences.

2. There was no effect of belimumab on the frequency of the autoreactive VH4-34 gene or on CDR3 length or composition in unmutated IgM sequences.

3. There was a significantly greater loss of VH4-34 among mutated IgM sequences and plasmablast sequences compared with unmutated sequences in subjects treated with chronic belimumab than in lupus controls.

Conclusions Although BAFF highly regulates survival of naïve $\mathrm{B}$ cells past the T1 stage in humans, we were unable to identify an effect of belimumab on $\mathrm{VH}$ distribution or CDR3 composition of naïve $B$ cells, suggesting a minimal effect on selection of the naïve B cell repertoire. By contrast belimumab may promote negative selection of autoreactive activated naïve $\mathrm{B}$ cells and plasmblasts.

\section{Al-19 T PERIPHERAL HELPER CELLS ARE EXPANDED IN THE CIRCULATION OF ACTIVE SLE PATIENTS AND CORRELATE WITH CD $21^{\text {LOW }}$ B CELLS}

${ }^{1}$ Deepak A Rao*, ${ }^{1}$ Alexandra V Bocharnikov, ${ }^{1}$ Chamith Y Fonseka, ${ }^{1}$ Joshua Keegan, ${ }^{2}$ Betty Diamond, ${ }^{3}$ Jennifer H Anolik, ${ }^{1,4}$ Peter A Nigrovic, ${ }^{1,5}$ Soumya Raychaudhuri, ${ }^{1}$ James A Lederer, 'Michael B Brenner. 'Brigham and Women's Hospital and Harvard Medical School, USA; ${ }^{2}$ Feinstein Institute for Medical Research, USA; ${ }^{3}$ University of Rochester Medical Center, USA; ${ }^{4}$ Boston Children's Hospital, USA; ${ }^{5}$ Broad Institute of Massachusetts Institute of Technology and Harvard University, USA

\subsection{6/lupus-2018-Ism.19}

Background Pathologic T cell-B cell interactions and production of autoantibodies are hallmark features of SLE. T follicular helper (Tfh) cells are generally considered the principal $\mathrm{T}$ cell population capable of helping $\mathrm{B}$ cells. However, distinct $\mathrm{T}$ cell populations can augment $\mathrm{B}$ cell responses in chronically inflamed peripheral tissues. We recently described a dramatically expanded population of $\mathrm{T}$ peripheral helper (Tph) cells that promotes B cell responses in synovium of patients with seropositive RA. Here we evaluate the frequency, phenotype, and clinical associations of Tph cells in the circulation of patients with lupus.

Methods Mass cytometry data from the Accelerating Medicines Partnership RA/SLE Network were used to quantify cell populations in PBMCs from 27 lupus nephritis patients, 27 RA patients, and 25 non-inflammatory controls. Frequencies of Tph cells (PD- $1^{\text {hi }} \mathrm{CXCR}^{-} \mathrm{CD} 4^{+} \mathrm{T}$ cells), Tfh cells $\left(\mathrm{PD}-1^{\text {hi }}\right.$ $\mathrm{CXCR}^{+} \mathrm{CD}^{+} \mathrm{T}$ cells), and $\mathrm{CD} 21^{\text {low }} \mathrm{CD} 19^{+} \mathrm{B}$ cells were quantified by standardized gating, and associations with SLEDAI and dsDNA titers were assessed. For in vitro T cell-B cell co-cultures, sorted Tph cells, Tfh cells, or control T cell populations from SLE patients were co-cultured with memory B cells and stimulated with SEB +LPS, and CD $38^{\text {hi }} \mathrm{CD} 27^{+}$plasmablasts were quantified at day 5 .

Results We first confirmed that Tph cells (PD-1 ${ }^{\text {hi }}$ CXCR5 ${ }^{-}$ $\mathrm{CD}^{+} \mathrm{T}$ cells) from SLE patients possess B cell helper function, as we previously observed in RA. Tph cells sorted from blood from 5 different lupus patients strongly induced B cell differentiation into $\mathrm{CD} 38^{\text {hi }} \mathrm{CD} 27^{+}$plasmablasts in vitro (figure $1 \mathrm{~A}, \mathrm{n}=5$ donors). By mass cytometry, Tph cells are markedly expanded in the circulation of SLE patients compared to non-inflammatory controls $(4.3$-fold increase, $\mathrm{p}<0.0001$, figure 1B). Tfh cells are also increased in the SLE patients compared to controls (1.9-fold); however, the magnitude of the increase in Tph cells in SLE patients well exceeds that of Tfh cells. Tph cell frequency is higher in lupus nephritis patients with dsDNA titers $>50(p=0.017)$ and with SELENA-SLEDAI $>10$ $(p=0.046)$ compared to patients with lower disease activity measures. Similar associations with disease activity were not observed for Tfh cells. Expression of surface receptors on Tph cells from SLE and RA patients was similar. A strong positive correlation emerged between the frequencies of $\mathrm{Tph}$ cells and CD21 $1^{\text {low }} \mathrm{B}$ cells, an activated B cell population highly expanded in SLE (Spearman $r=0.56, p=0.0026$, figure $1 \mathrm{C}$, gray $=\mathrm{SLE}$ patients, black $=$ all other patients). In contrast, no correlation was seen between Tfh cells and CD21 $1^{\text {low }} \mathrm{B}$ cells in SLE patients.

Conclusions Tph cells are markedly expanded in the circulation of patients with SLE and demonstrate robust B cell 
A

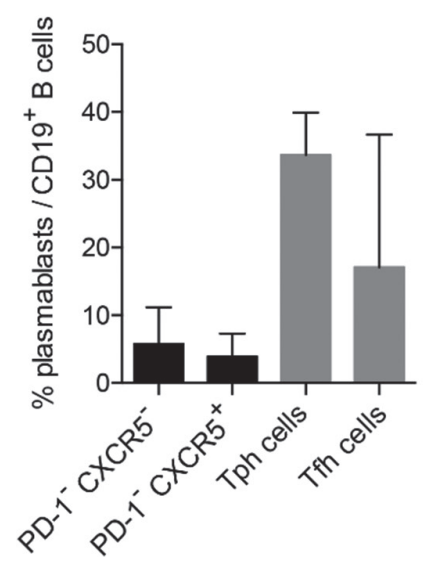

B

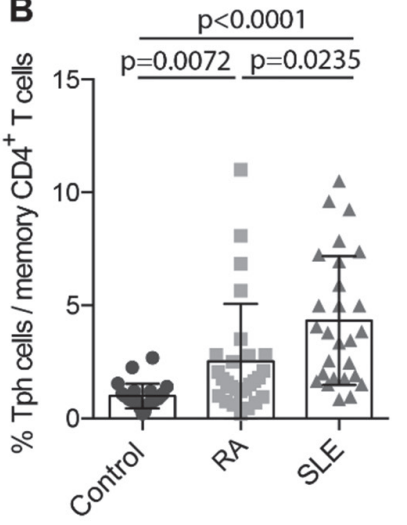

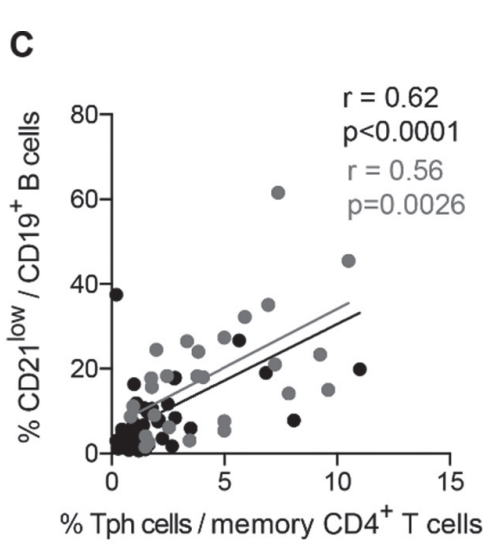

\section{Abstract Al-19 Figure 1}

helper function. The strong and specific positive correlation between Tph cell and CD21 $1^{\text {low }} \mathrm{B}$ cell frequencies suggests that these cells may act coordinately in the pathologic autoimmune response in SLE.

Acknowledgements We acknowledge the Accelerating Medicines Partnership RA/SLE Network and its members.

\section{Auto Antibodies}

\section{AA-01 PHENOME-WIDE ASSOCIATION STUDIES UNCOVER HIERARCHY OF AUTOANTIBODIES IN SYSTEMIC LUPUS ERYTHEMATOSUS}

${ }^{1}$ April Barnado*, ${ }^{2}$ Robert J Carroll, ${ }^{3}$ Carolyn Casey, ${ }^{1,2}$ Joshua C Denny, ${ }^{1}$ Leslie J Crofford. ${ }^{1}$ Department of Medicine, Vanderbilt University Medical Center, USA; ${ }^{2}$ Department of Biomedical Informatics, Vanderbilt University Medical Center, USA; ${ }^{3}$ Department of Medicine, Lehigh Valley Health Network, USA

\subsection{6/lupus-2018-Ism.20}

Background Systemic lupus erythematosus (SLE) is a heterogeneous disease with diverse presentations. dsDNA antibodies associate with renal disease. Less is known about comorbidities in SLE patients without dsDNA antibodies. Using a large electronic health record (EHR) cohort, we sought to identify comorbidities that associate with dsDNA status. We used a technique that scans across EHR billing codes called phenomewide association study (PheWAS) to compare SLE patients with and without dsDNA antibodies. We also evaluated the relative importance of SLE specific autoantibodies on SLE criteria.

Methods We used our validated SLE algorithm of $\geq 4$ counts of the SLE ICD-9 code and ANA positive $\geq 1: 160$ while excluding dermatomyositis and systemic sclerosis ICD-9 codes with a positive predictive value of $94 \%$ and a sensitivity of $86 \%$. We identified SLE cases in a de-identified EHR that contains over 2.8 million subjects. Autoantibody status was defined as ever positive for dsDNA, RNP, Smith, SSA, and SSB. PheWAS was performed in dsDNA positive vs negative SLE patients using logistic regression adjusting for current age and race. For multiple testing, a false discovery rate (FDR) $p$ of 0.05 was used. We also performed logistic regression to assess the impact of autoantibodies, age, sex, and race on SLE criteria.

Results We identified 1097 SLE subjects. As expected, dsDNA positive subjects, compared to dsDNA negative, were more likely to have renal codes including nephritis $(\mathrm{OR}=4.60, \mathrm{FDR} \mathrm{p}=2.33$ $\left.\times 10^{-9}\right)$, renal failure $\left(\mathrm{OR}=2.30\right.$, FDR $\left.\mathrm{p}=1.85 \times 10^{-5}\right)$, and end stage renal disease $(\mathrm{OR}=2.63, \mathrm{FDR} p=0.01)$. After adjusting for sex, race, age, and other autoantibodies, dsDNA was independently associated with nephritis $\left(\mathrm{p}=1.98 \times 10^{-6}\right)$ and chronic kidney disease $(p=0.001)$ and also associated, along with SSB, with serositis $(p=0.01)$ and hematologic criteria $(p=0.001)$ (figure 1). dsDNA negative subjects were more likely to have codes for sleep, pain, and mood disorders.

Conclusion Using PheWAS, we uncovered a hierarchy within SLE specific autoantibodies where dsDNA, compared to other autoantibodies, was a powerful predictor of major organ involvement in SLE.

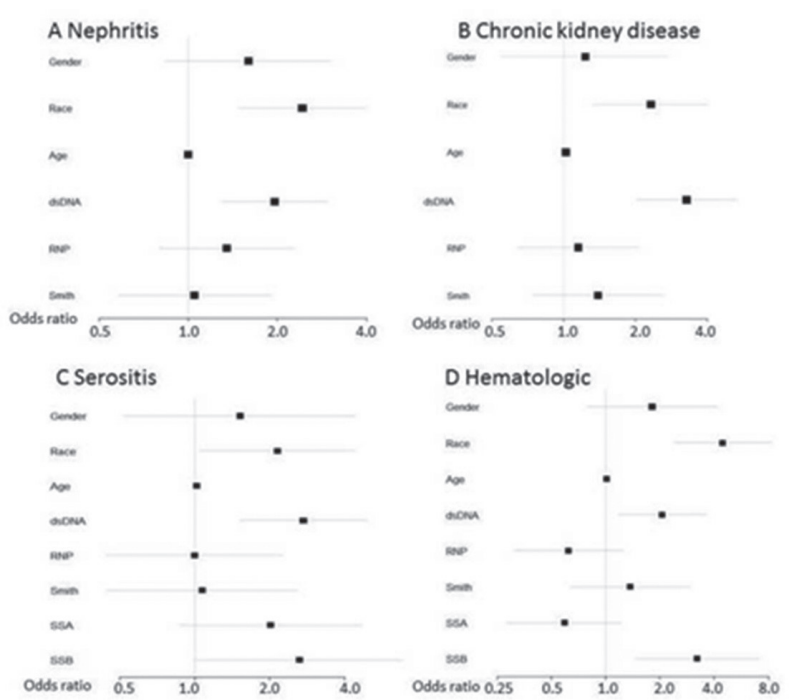

Abstract AA-01 Figure 1 Forest plots of logistic regression models for SLE criteria. Logistic regression models for nephritis (A) chronic kidney disease (B) serositis (C) and hematologic criteria (thrombocytopenia, pancytopenia) (D) were created with covariates shown on the left. Odds ratios are across the bottom with horizontal lines depicting $95 \%$ confidence intervals. 